\section{RATIONAL THERAPEUTICS AND THE USE OF COMMON REMEDIES. ${ }^{1}$}

BX SAMUEL WEST, M.D. OXON., F.R.C.P. LOND.,

AGSISTANT PHYSICLAN AND DEMONSTRATOR OF PRACTICAL MEDICLNE A' TrREE HOSPITAL, HTC.

Threge is, I think, no better work that we can do as practitioners of medicine than to meet together from time to time in a friendly and informal manner in order to discuss the drugs we are prescribing every day and to compare notes of our experience as to their use. Therapeutics has not yet passed out of the empirical stage, and from whatever source the suggested remedy comes its value has to be tested and proved at the bedside. It is to empiricism tested by bedside observation that medicine owes most of the remedies it finds of use. Yet we often hear this empirical bedside study of the action of remedies spoken of with disparagement, as if it were the opposite of rational, and it was a protest against this misuse of terms that formed one of the most striking passages in Dr. Gowers' recent address. Essentially false as the opposition between empirical and rational really is, it is based upon a laudable desire to bring therapeutics into closer relation with the general principles of science. Unfortunately, this is but a pious wish and not yet an achieved fact. the same confusion between a wish and a fact finds expression in another form in the contract that is often made between pharmacology and therapentics.

Pharmacology is a word of recent introduction. Ending in "logy" it is assumed to be a science, and, being a new word, to denote a new science. Now, it will be well to see how far this claim is just. I take down from my shelf some recent book which bears upon its back the title "Pharmacology," and another, written but twenty years ago, labeled "Therapeutics." I turn up in both the same drugs, and what do I sind? First, an account of the crude substance and of the sources whence it is derived; then an account of its chemical constitution, of the methods by which the substance can be obtained, the impurities which are present, and the way in which they may be detected and removed; next the results of the physiological investigations into the action of the drug, for the most part performed in the physiological laboratory, upon lower animals, with a - summary of the conclusions to be drawn from these experiments and the suggested application to their use in disease; and in all these respects there is no greater difference between the two accounts than the lapse of time and the progress of knowledge accounts for. But the greater part of the account in each case alike is formed, as would be expected, of a statement of the effect of the drugs upon man in health and in various conditions of disease. This part forms threequarters, nine-tenths, or in some cases even more, of the whole. And how has it been obtained? By clinical observations such as you and I can make and are making every day. If, then, we take away from the account given of any remedy the materia-medical, therapeutic, and toxicological parts, there is notbing left for pharmacology but the physiological action of the drug upon animals, in which special subject of recent years great advance has been made. In this sense pharmacology as distinguished from what used to be called therapeutics comes to be nothing more than a special department of Applied Phy:iology.

The term is, however, often used in a less restricted sense, and taken in a general way to mean all methods of treatment and the explanations to be given of their mode of action. Thus of quite recent modern books on this subject some, like Ringer's in this country and Wood's in America, are entivled "Therapeutics"; others, like Brunton's, "Materia Medica, Pharmacology, and Therapeutics" while others, especially on the Continent, bear the simple title of "Pharmacology." Yet there is no difference in the contents of any of these books, the difference lies all in the title-Pharmacology; this proves to be, in fact. but a new name for the old Therapeutics-Rationalistic Therapeutics, if we like to call it so-in which all the sciences, chemistry,

1 An introduction to a discussion, read before the Willesden and District Medical Society on Jan. 17th, 1896 physics, physiology, and pathology, are called into requisition to give or to suggest explanations of the results at which clinical observation has arrived. This double use of the term is responsible for much confusion and misconception. The most serious differences between men arise, not so much about things or facts, but because of words the meanings of which have not been accurately defined; and so it is with pharmacology. At the present time this matter has a practical bearing, for everywhere examinations for medical students are being instituted in this subject, and it becomes therefore necessary to define exactly what is meant. We should all agree that we ought to send our students out into practice as highly equipped as possible with therapentic knowledge and to train them so that they should be able, not only to practise therapeutics, but to think and reason about the ways in which their remedies act, and this knowledge must be tested by examination; but it does not follow that an examination in a special department of applied physiology is also necessary for the purpose. Indeed, the requiring of such examinations seems to rest upon the assumption that therapeutics is an art based upon a science of pharmacologyin other words, that therapeutics is applied pharmacology. Put in this form the fallacy is obvious. It is an absolute reversal of the real relation between the two subjects at the present time, for even the most recent works on pharmacology contain, as stated, comparatively little beyond that which has been derived from empirical therapeutics. We trust this will not always be so, but it cannot be denied that this is the case at the present time.

This subject has a special bearing upon the present discussion, for none of us, I presume, claim to be pharmacologists in the limited sense of pharmacological physiologists, yet we are all therapeutists, and, I believe, thoughtful therapeutists. We use the remedies which experience recommends, and must therefore, I suppose, be considered empirics. Yet we bring to bear all our powers of clinical observation to determine the conditions under which these remedies act, and we try to find if we can some scientific reason why they produce their result. If we can get good reasons we are glad; if not we continue at any rate to use the remedies which we know by observation prove beneficial. If this is not rational treatment, what is it? We may at any rate claim to be rationalistic therapeutists. The real difference between a pharmacological physiologist and a clinical therapeutist is but one of method and of place. Each has an equal desire to know how remedies act and each attempts to solve the problem in his own way. The objects are the same, the methods different. Each field of research is necessary to the other, and each real worker knows it. The special line of research is determined by the man's opportunity and his taste. The physiologist investigates his remedy in the physiological laboratory upon animals, and he has the great advantage that he can modify his experiments at will and make them at his own time and in his own way. The clinical therapeutist's laboratory is the sick room or hospital ward; the subject of his experiments is man, not healthy man, but sick; the conditions of experiments he cannot vary for himself at will; he must take the patient as he is presented to him, at a time which he cannot choose, and when he is perhaps least inclined or fitted for such investigation. But the greater the difficulty the greater the honour of achievement, and I think no one will deny that the difficulties of the clinical investigation of the action of drugs are out of all proportion greater than those which attend physiological investigation. It is perhaps in their suggestiveness that the physiological and chemical studies of remedies have their chicf value. For instance, how suggestive is the theory that substances with like chemical constitution bave similar physiological action, and what a field of research does it seem to open out. To what brilliant results are Pasteur's observations on fermentation and the lower organisms already leading. Yet is not bedside observation rich in suggestions too? Can we afford to discourage, slight, or despise them because they come from another source than pure science, those happy thoughts, ideas, inspirations we might almost call them, seeds which chance and casual observation dropped into an observant mind? Is not Jenner's discovery of vaccination receiving at last the scientific explanation in Pasteur's work, and shall we call Pasteur's work rational and deny the term to Jenner's?

Yet, after all, wherever the idea comes from, whether from the chemist, physiologist, pathologist, or busy practitioner, in all cases alike, it is at the bedside that it must be tested. This is, after all, the crucial test, whatever the theory 
be. Many remedies we use and cannot tell why they do good, though we much wish to know; experience proves their value, therefore they must be used. On the other hand, many remedies which come to us rich in scientific promise put to the test prove not to fulfil the promises they held out. Surely it would be as irrational to discard the first as to continue to use the latter.

Rational therapeutics consist in the use of remedies which we know to do good, whether we seem to understand their mode of action or not. But before we endeavour to explain a result we must know whether it is a fact or not, otherwise we are but beating the air. This constant testing and proving of stated facts is the chief function of the therapeutist and for the carrying on of certain lines of such research those have great advantages who are attending patients in their own homes. On practical points of this kind it is that discussions such as that which I have the honour to introduce are likely to prove full of instruction and profit.

The drugs which have been selected for consideration are five: iodide of potassium, liquor arsenicalis, tincture of digitalis, liquor strychniæ, and antipyrin. I propose to take them one by one, for in this way I think the discussion will be more precise and profitable.

\section{IODIDE of POTASSIUM.}

The first on the list is iodide of potassium and this drug illustrates very well the evolution of the science of therapeutics. Crushed sponge was known as a successful remedy for goitre in the thirteenth century and it is probable that it was used by the Chinese much earlier. Fucus vesiculosus was also used later for the same purpose. Iodine was discovered by Courtois in 1811. In 1820 Coimdet of Geneva, finding that both these remedies contained iodine, suspected that iodine was the active agent in the cure, and his theory when put to the test proved to be correct. He extended its use to the treatment of scrofulous glands, and then came the discovery of its action in syphilis in 1831 and of its effect in the elimination of lead and mercury from the body in 1844 . Further investigation has shown that iodide of potassium is eliminated chiefly by the kidneys, but it is found in the blood, saliva, milk, and even in the urine of a sucking child of a mother who is taking the drug, and that it is present also in the skin, liver, spleen, lymph glands, and muscle, but not in the brain. Its physiological action is not understood, and nearly all our knowledge of the drug is derived from the result of clinical observation-i.e., it is simply empirical therapentics.

Syphilis.-_On the use of iodide of potassium in syphilis there is no need to dilate. The rapidity with which tertiary lesions, such as periosteal nodes, skin eruptions, and iritis, disappear under its action are among the triumphs of therapeutics. Take again a patient with third nerve paralysis. What a comfort it is to know that as such a lesion is almost always syphilitic it will get well under iodide of potassium. In the early stages of syphrlis the diagnosis is usually easy, but it is in the later stages that difficulties of diagnosis begin. It is then that diseases of the nervous system and of the viscera prevail. It is then that nondescript nervous symptoms develop: pains in the joints, pains in the bones and head, over the liver and in the abdomen, and even in the chest; perhaps paroxysms of intense neuralgia. Treatment on general lines has been tried without success until the syphilitic origin is at last suspected, and iodide of potassium effects the cure. Perhaps some of the most striking cases are those in which profound cachexia develops without obvious cause, a condition common enough in children and generally recognised, but not rare either in the adult. I saw a young woman some time ag' who was healthy until marriage, and although she had harl no sign of syphilis, and her medical attendant could not believe that she had been syphilised, yet her husband was known to have led a loose life, and iodide of potassium restored her to health when iron and all other treatment had failed. I saw a man not long ago with symptoms which might have been due to advanced multiple sclerosis or even, except for the want of mental symptoms, general paralysis of the insane, in whom no history of syphilis could be obtained, yet iodide of potassium and rest rapidly cured him, and the case was doubtless of a syphilitic nature. Of the cure of syphilitic diseases of the nervous system by iouide of potassium we must speak with caution, yet all will admit the benefit the drug does, though the question of complete cure may be more difficult to answer. I do not think we can go so far as to say tbat the cure of a doubtful disease by jodide of potassium is certain proof of specific origin, but in many cases it certainly is strong presumptive evidence. No doubt much depends upon the stage of the disease. The most advanced forms of syphilis of the nervons system consist in fibroid degenerations, and we can no more expect to remove these by drugs than we can to charm away the scar of the syphilitic ulcer in the skin. Yet in the early stages of sypbilitic infiltration we may expect to do, and really probably succeed in doing. as much as we see daily happen under the action of the rlug in the skin and superficial parts of the body. It is in the early stages of syphilifie disease of the nervous system, when the lesions are recent and gummatous, that cure may be effected, not in the later, when irreparable damage has been done and the wasted nervous structures have been replaced by fibrous tissue. It is in these stages, when the nervons affection can hardly be definitely named and before it can be called, e.g., tabes dorsalis or lateral sclerosis, that the good may be done and, as in some of the cases mentioned, apparent cure be the result of treatment. It is usually stated that in cases of syphilis which have been already treated with mercury iodide of potassium converts the insoluble compourd which is supposed to be formed between mercury and the tisstes into a soluble one, thus setting the mercury free again, and it is in this way that the salivation sometimes cansed by iodide of potassium in these cases is explained. It is, how. ever, proved that iodide of potassium has a curative effect on syphilis in a person who has taken no mercury at all. It would be interesting to hear of instances of this kind, for in the present day, when syphilis is treated with mercury as a matter of routine, such cases are necessarily rare.

Poisoning nith lead and mercury. - There can be no doubt of the effect which iodide of potassium bas in promoting the elimination of these two metals in cases in which symptomy have been produced by them. Clinical experience is eonfirmed by the chemical examination of the urine in which the administration of the drug is increased by an increased elimination of the metal. These metals form insoluble compounds with albumin and thus become fixed in the tissues. Iodine releases them and does so, it is supposed, by forming a soluble substance by double combination with the metal and albumin. This pharmacologictl explanation even if it proved to be incorrect would not affect the correct ness of the clinical observations.

Aneurysm.-A great deal has been written abont the efficacy of iodide of potassium in aneurysms, especially intra-thoracic aneurysms of large size, and I think there can be no doubt that it does relieve the pain and lead to diminution in the size of the sac, yet it is not easy to determine this point because the patient is usually at the same time being treated in other ways-e.g., by diet and complete rest. Certainly the improsement is not due simply to coagulation in the sac, for in many cases where much relief has been experienced the sac has been found empty of clot. It is tempting to believe, inasmuch as so many aneu ysms are the result of syphilitic disease of the arteries, tbat iodide of potassium might cure them; bnt whatever action it might have upon early syphilitic disease of the vessels (and of this there can be little doubt) it can hardly be expected to produce any effect when a large aneurysm has formed, for the aneurysm is the consequence of past syphilitic disease and not associated in all probability with any active disease of the kind. The accepted explanation is that the iodide of potassium reduces blood pressure and diminishes the force of the heart's contraction, the aneurysmal sas being thas permitted to contract and the symptoms being rtiever.

Rheumatism.-Iodide of potassium is one of the stock remedies for all forms of muscular rheumatism, though its place is being taken to a great extent now by salicylate of soda. In the same way it is one of the stock remedies for all forms of the chronic joint troubles which are called by the generic term of "rheumatism." It is, however, entirely without effect in rheumatic fever, a specific affection which in my opinion, has nothing whatever to do with the great majority of cases which we call rheumatism in this country. Its effect is most striking perhaps in those cases of chronie rheumatism or gout which are associated with chronic lead poisoning.

Diseases of the chest.-In the treatment of diceases of the chest iodide of potassium does not play any great part. It seems to be of service in some cases of chronic bronchitis. possibly because it is excreted by the mucous membrane of the bronchi, as it is by all other mucous membranes. In 
asthma it certainly is a remedy of use, not so much at the time of the paroxysms as between the attacks, and it appears to have the effect of diminishing their frequency. Certainly, many asthmatic patients believe in the drug and attribute a great benefit to the taking of it. In plastic bronchitis it is said to be the only remedy which is of any service at all, and so far as my experience goes, which now extends to several cases, I certainly endorse that opinion.

Iodide of potassium appears to be an alterative-i.e., to have a subtle influence upon cell nutrition. For this reason it is largely given in the chronic processes of inflammation, with a view of controlling it or of removing its products. It is thus one of the chief routine remedies for almost all chronic inflammatory affections of the joints, especially of the serous membrane-e.g., of the joints and pleura. As an alterative also it is given in all forms of enlargement of the lymphatic glands, especially those of a tuberculons nature, and it is stated to have a strong action upon certain other glands, especially the testicle and the mamma; thus it is an efficient galactogogue, while prolonged administration of the drug may, it is stated, lead to atrophy of the testicles. It will be interesting to have any confirmation of these statements, of which I have no personal experience to bring forward. Upon dropsy it has been said to have a specific effect, but there are so many causes of dropsy, and the term is such a loose, indefinite one, that it is hard to know exactly what is meant. In diseases of the skin it is not much employed, unless in those of a syphilitic nature; but I have seen it do much good in large doses in scleroderma.

I now come to a very interesting part of my subjectviz., the consicleration of the peculiar effects which are sometimes produced by the administration of the drug ; and here we are brought face to face at once with one of the most disturbing factors in therapeutics-viz., idiosyncrasy, that great stumbling block in the way of applying any physiological results upon animals to man or even without caution to all men alike. Iodism in its ordinary form of headache and catarrh I need hardly do more than mention. I suppose we may assert that it is capable of being produced in every patient, yet there are striking differences in this respect following in one case the administration of the smallest dose, while in others not appearing until very considerable doses have been taken for some time. As iodism is one of the practical difficulties we have to deal with in the administration of the drug it will be well, therefore, to consider what means there are of obviating it. It is in many patients a merely transitory phenomenon, and after two or three days the symptoms pass off, though the dose has not been changed, and even if the dose has been increased; indeed, it has been paradoxically asserted that the cure for iodism is to double the dose. The subsidence of symptoms is aided often by the administration of a saline purge. Usually if the medicine be reduced or stopped for a day or two until the symptoms have passed off its administration may be then resumed and iodism will not return; but in some cases the idiosyncrasy is so marked that even the smallest dose produces the effect, and nothing short of the entire giving up of the drug will suffice. But before this it will be well to try whether the iodide of ammonium or of soda is not tolerated better. It is stated that the addition of carbonate of ammonia or of the aromatic spirit of ammonia to the mixture will check the occurrence of jodism, but I have failed to satisfy myself of this, and so I see has Ringer. Another form of iodism, though not quite so common as the eatarrhal form, is that in which the drug causes great depression of mind and body, often without coryza or any other of the common symptoms. Sometimes it upsets the digestive organs, causing loss of appetite, nausea, vomiting, or watery purging. Puffiness and swelling beneath the eyes are not uncommon with marked coryza, but they occasionally occur without it, thus giving the face a renal aspect. Salivation and diaphoresis are also stated to be occasional results, but I cannot say that I have observed them.

The iodide eruptions have always excited interest. The commonest of them is acne, and this occurs in the usual places, but the acne spots are often of considerable size and attended with a good deal of inflammatory induration. Upon the face, usually associated with acne, a butterfly eruption is often seen, of an eczematous type, extending beneath the eyelids and across the bridge of the nose, and in most of these cases there are well-marked acne spots on the lower part of the nose and cheek. The most striking iodide eruptions, and those most commonly meant by the name, are those which are pustular or even bullous in character, and their usual seat is the face; and, strange to say, some of the mos marked forms of eruption have appeared some time after the administration of the drug has been suspended. General diffuse erythema is met with sometimes over the whole body, but it is stated to be rare. I do not think it is so uncommon on the legs, and in this position it is often hæmorrhagic. A patient came under my treatment for a punctate hæmorrhagic erythema of the leg and he volunteered the statement that, among the many things that would produce it, iodide of potassium was the principal. I was subsequently enabled to observe that the administration of a few grains of iodide of potassium brought out markedly hæmorrhagic erythema. Lastly, cases of general purpura have been described, and one or two of these have proved fatal, but this condition is extremely rare.

The effect of iodine upon the lesions of syphilis has led to its use in other specific affections, especially, for instance, tubercle. In tuberculous affections of the lymphatic glands, as well as in tuberculous affections of the peritoneum and perhaps of the pleura, it does seem to have some effect. In phthisis and in tuberculous peritonitis I believe it to be useful. There is one other rare but remarkable disease in which it seems to be by far the most effectual remedy we possess: I refer to actinomycosis. In a little patient recently under my care suffering from this disease involving the pleura and chest-walls, the child, who had been reduced to a shadow and looked desperately ill, made no improvement until iodide of potassium was administered, and then at once gained flesh, colour, and strength, and seemed as if he might recover. Unfortunately, he died by misadventure during the administration of chloroform given for the purpose of opening one of the small abscesses.

The therapentics of iodide of potassium open out a very large field of discussion, but there are, I think, one or two points upon which information would be specially valuableviz , (1) iodism and the means of counteracting it ; (2) iodide rashes and the way to remove them ; (3) examples of peculiar idiosyncrasies; (4) instances in which the drug has caused stoppage of the secretion of milk or atrophy of the testicles, free purgation, or vomiting; and (5) instances in which effects have been produced upon a suckling child through the milk of the mother while taking the drug.

In respect of the mode of administration it is usually given in solution in water or it may be given in milk. One point has come out in recent years-viz., that very large doses of the drug may succeed in producing an effect when small ones have failed, and the amount so given in the twenty-four hours sometimes reaches extraordinary dimensions, even to the extent of four or five ounces.

\section{LIQUOR ARSENICALIS.}

The next remedy on the list is liquor arsenicalis. The history of arsenic in therapeutics is interesting from our present point of view. It was known to the ancients in the form of orpiment and sandarach or realgar. Dioscorides gives the following description of the drug: "Orpiment, auri-pigmentum-i.e., the yellow sulphide-is astringent and corrosive, burning the skin with severe pain with the production of an eschar. It removes fungous flesh and is a depilatory." He then describes sandarach or realgar-the red sulphide -in the same terms, stating that, "mixed with resin, it causes hair to grow when destroyed by pellagra, and that with pitch it removes rough and deformed nails. With oil it is used to destroy lice, to dissolve abscesses, to heal ulcers of the nose (probably lupus), of the mouth, and fundament (probably epithelioma). With wine it is given for fetid expectoration, and its vapours, with those of resin, are inhaled in chronic cough. With honey it clears the voice and with resin is very excellent for shortness of breath." In China it was used from very early times as an application to venomous wounds, also as a remedy for ague and as a tonic in several diseases. For skin diseases it has been employed by the Hindoos for centuries, though its use was not introduced for this purpose into this country until the end of last and the beginning of this century. It was introduced into Europe about the beginning of the seventeenth century, and was then used as a remedy for pulmonary affections associated with dyspnca, probably asthma and chronic bronchitis, as well as for different forms of ague. The analysis of a patent ague medicine by Fowler, and the discovery in it of arsenic, led to its extensive use for intermittent fever in this country; and at the end of last century it was a common remedy also for various scaly skin eruptions and for neuralgia. After having had a period 
of popularity it fell into disuse and was almost forgotten until Boudin, in the middle of this century, resuscitated it, and since that time it has been one of our stock remedies for all the affections named. Of recent years numerous experiments have been performed on animals, and they seem to show that arsenic has a definite effect upon the nervous system, especially upon the cardiac ganglia and respiratory centres; but it is difficult to see any relation between the results of these experiments and the action of the drug in man; so that we are indebted again for our knowledge of the action of this drug in disease simply to observation upon man -in fact, to the oft-disparaged empiricism, or, as I should prefer to call it, to thoughtful clinical observation. I do not propose to speak of the local action of arsenic externally, but only of its internal use as a physic; and this limitation, I believe, the committee desired, when they placed upon the list they first sent me, not "arsenic," but "liquor arsenicalis."

The facts about the drug other than clinical which have been discovered by clinical and pathological investigation are these: that arsenic is rapidly absorbed from the stomach and found in a few minutes in the circulation; that it is eliminated chiefly by the urine and sweat, and also by the mucous membrane and glands; but the elimination takes place slowly, so that arsenic may be detected a fortnight or more after it has been stopped. It is also eliminated by the liver, and in full doses causes fatty degeneration of both the liver and the kidneys, with the occurrence of albuminuria. It has especial affinity for the nervous system and is found in very much larger amount in the brain and spinal cord than in the other tissues of the body, except, perhaps, the liver. In toxic doses, besides its effect upon the liver and kidneys, it destroys the red cells, dissolves the hæmoglobin, and as the result leads to hæmorrhages into the various tissues of the body.

Ague.-Arsenic is now Frecognised as the most powerful anti-periodic except quinine, and it may succeed where quinine has lost its power, and it is stated that the relapses are longer delayed under treatment by arsenic than by quinine. It is useful also in all affections of malarial origin, and especially in those malarial neuralgias the causes of which are often so difficult to determine. I do not know that any satisfactory explanation of its action in ague has been given, yet it may lie in the action of arsenic on the blood-cells in which the parasite of ague is found.

Diseases of the skin.-It is one of the stock remedies in all the chronic diseases of the skin, and especially in psoriasis. In some cases of chronic eczema it is found useful, but in many its use is disappointing, and in some it does positive harm. It is largely used in animals to give them a fine glossy coat, and in Styria and the Tyrol it is habitually taken by both men and women to produce embonpoint and fineness of complexion. Of recent years it has been largely advocated for the treatment of herpes zoster and post-herpetic neuralgias, but its use, I think, is disappointing.

For asthma it is a very old remedy and in certain cases almost a specific, being administered either by the mouth or by inhalation, as in the form of cigarettes. As a general tonic it is useful in a variety of conditions, provided it does not upset the stomach, but this can be generally avoided by giving it in small doses after food. Of recent years it has been largely used in affections of the nervous system, possibly because of the affinity which the drug shows, as already stated, for nervous tissues. Thus it has been given in epilepsy, but I think without much success. For neuralgias of all kinds it is an effective remedy. It is said to do good even in attacks of angina, but of this I am sceptical; but, having other more reliable remedies, I admit that my scepticism does not rest on much experience. As already stated, its chief use is in malarial neuralgias-e.g., brow ague.

The nervous disease for which it is most employed at the present time is chorea. It used to be recommended that small doses should be given and continued for some time. The result was not very obvious. Recently large doses have been advocated as soon as the patient comes under treatment, even as much as fifteen or twenty minims of the liquor arsenicalis three times a day even to a small child. It is often easy, if the drug be gradually increased, to reach amounts like this in the adult as well as in children, but it is somewhat startling to begin with such large doses from the first. However, I have myself tried this treatment in a number of cases and I have not seen any poisonous effects produced by it. Yet I cannot say that I am satisfied as to the immediate benefit which follows, nor can I endorse the statement that large doses will cut short a bad case within a few days. In a severe case of chorea which has been neglected and badly treated at home admission into a hospital is sure to be followed within a few days by marked improvement without the use of drugs at all, and if arsenic be administered as soon as the patient is admitted it becomes extremejy difficult to know whether the improvement is to be referred to the drug or to the general management of the case. In private practice, where the cases are more closely under observation, it is quite possible that valuable information on this point may be forthcoming.

I do not propose to go into the question of acute arsenical poisoning, accidental or intentional. But on the subject of chronic arsenical poisoning of accidental origin, such as that acquired by arsenical papers and in other methods, a society of this kind would be likely to give information of great value and interest. The chief symptoms are general failure of health, loss of appetite and impaired digestion, with weakness of the eyes, thirst, salivation, cramps and colic. and in extreme cases emaciation, edema of the face and body, perhaps albuminuria and ulceration in the skin. When symptoms of profound failure of health of this kind arise, for which no cause can be found, it is well to bear in mind the possibility of some accidental poisoning, of which, perhaps, arsenic is the most likely, sometimes accidental, as in the case of arsenical papers, sometimes intentional, with criminal intent, as in certain legal cases which we can all remember. Tolerance of the drug is easily established, so that the prolonged administration of the medicine necessitates the increase of the dose. The amounts which may be taken may be very large, as with the arsenic-eaters of Styria and the Tyrol. Catting off the drug suddenly in such cases is said to produce grave symptoms of poisoning, which may be relieved when the drug is resumed. It would be interesting to know if any experiences of arsenic taking and its results can be communicated.

The prolonged administration of the drug is sometimes followed by mischievous, though very interesting, results, of which I may mention two-viz, arsenical pigmentation and peripheral neuritis.

Arsenical pigmentation is comparatively rare. Its colour is a dull brown. It may be general, but is more often irregular in distribution and sometimes more marked on exposed parts-e.g., the face and neck. When given to cure a skin disease - e.g., psoriasis - it may be limited to the seat of the eruption. Other skin eruptions are rare. Papular, measlelike rashes are described on the face and other parts of the body, and urticaria is comparatively common. Herpes zoster has been attributed by many writers to the drug, and pustular ulceration or even gangrenous eruptions have occurred after toxic doses. In these rashes the effect of idiosyncrasy is strongly marked. Thus marked pigmentation followed the taking of three minims only of the liquor arsenicalis three times a day for a week only, and in another case five minims had been taken three times a day for three weeks. Neuritis following arsenic is very rare and all cases of it should be recorded. It resembles lead poisoning and may appear as wrist-drop, though in advanced cases it may more closely resemble alcoholic neuritis and affect both legs and arms. ${ }^{2}$

\section{TINCTURE OF Digritalis.}

Digitalis is a drug that has no ancient history. It was used in the sixteenth century as an application to wounds, a vulnerary, and in the middle of the seventeenth century Culpepper writes that "it has been used by the Italians as a dressing for recent wounds and old sores, that it is recommended as a purgative, emetic, and deobstruent, and that it is beneficial in scrofula and scalded head, and that it has been found of late to cure divers of the falling sickness that have been troubled with it above twenty years." It was introduced into the London Pharmacopoia in 1721, and again omitted in that of 1746 . It had been a popular remedy for some time in several parts of England for dropsy, and in 1775 was established as a good remedy for this condition by Withering, so that in 1788 it became a recognised article again in the Pharmacopoeia. From this time it has been largely used in clinical medicine, but the more exact study of the drug dates from the middle of the present century. The investigation of its physiological

2 Dr. James Taylor, in the course of the discussion, referred to the occurrence of neuritis following the administration of large doses of the drug in chorea. 
action shows that in animals small doses increase the contraction of the heart, while in large doses the heart becomes irregular and finally stops in a condition of contraction. It appears to stimulate the cardiac and vaso-motor nervous system, as well as to increase the force of muscular contraction.

In man it first produces slowing of the pulse with a rise of blood pressure, regulating the heart's action and strengthening it. This sedative and regulating action upon the heart caused Bouillaud to designate it "the opium of the heart." With 3arger doses the pulse becomes rapid and the pressure falls, and in toxic doses the pressure falls still more, the heart becomes slow, and more and more irregular until it finally stops beating.

In therapeutics digitalis is chiefly used on account of its effect upon the heart, and especially where the beart's action is irregular and weak, for which condition it bas become one of the stock remedies. Inasmuch as digitalis prolongs diastole and increases ventricular contraction it is just in mitral stenosis that it would appear to be chiefly indicated, for the lengtbening of diastole would give the blood longer time to make its way through the narrowed mitral orifice, while the increased ventricular contraction would drive a larger quantity of blood out into the arteries. thus improving the general nutrition of the body, in which the heart would also share. It is, in fact, in mitral stenosis that it is found to be most serviceable, but it is almost as useful in mitral incompetence too, and this does not seem quite so easy to explain. These theoretical considerations would suggest that it was distinctly contra-indicated in aortic regurgitation, and it is true that it has to be given with greater caution in all aortic cases; yet, with caution and in smaller doses, I am satisfied of its usefulness even in aortic regurgitation. It has been stated that it is not a desirable remedy in cases of fatty heart. I do not agree with this, for $I$ have given the drug frequently in all forms of weak heart and with great advantage, iron and digitalis being the most effective combination I am acquainted with for these conditions. It is especially useful in those cases of dilated and weak heart which are not uncommon in advancing years, but it is equally beneficial in other forms of weak heart, as, for instance, in the later stages of granular kidney, or even where the heart is enfeebled in the course of fevers.

In a very remarkable group of cases, that of acute myocarditis, of which I recorded an interesting series some Jears ago, the drug was very useful, but it exhibited in a marked degree the effect of the prolonged administration of the drug. At the time of commencing the treatment the heart was making a number of feeble beats, 150 or more in a minute, and digitalis was given with the view of decreasing their number and increasing their force. This it effectually did, and then after a time suddenly, without any symptoms at all in the patient to draw attention to the change, the pulse-rate fell, quite abruptly in some instances, to not more than 40 in the minule. The patient seemed in no way worse for this, and it would not have been discovered if the pulse lad not been felt and counted. The drug was stopped, some stimulants given, the pulse rose to 70 or 80 , and conxalescence proceeded satisfactorily. In most of the cases, rrave as the disease was, recovery took place but slowly and after a prolonged illness, as would be expected. Digitalis is also of great service in the alcoholic heart-i.e., the degenerate, dilated heart of drunkards, what is described popularly in Germany as the Bier-herz; but in this country we see it more often in spirit-drinkers. As a diuretic
digitalis acts not directly upon the kidney, but indirectly through the circulation by its effect upon the heart and vessels. In the early stage of acute renal disease, remedy, yet it is not infrequently prescribed, though for a different reason. It is given when the urine contains some considerable amount of blood, with a view of checking the hæmorrhage, but I do not think it a useful remedy even then. It is in the later stages of acute nephritis or in chronic parenchymatous nephritis, when the tension is low, the powers feeble, and the heart weak, that digitalis does most good, and then it acts by bracing up the circulation. In granular kidney, again, it is not until the later stages, when the tension falls and the heart is weak, that digitalis is a remedy of real use ; then it does good, for when the tone of the vessels is restored the patient is better. High tension in granular kidney is a part of the disease, and though a man would be better without a granular kidney at all, still if he has one he is better with high than low tension in his arteries, and it is, I believe, almost as great a mistake to use depressing remedies to lower tension in the early stages of granular kidney as it would be to refrain from giving digitalis, on theoretical grounds, in the later stages.

Digitalis has been advocated as a hæmostatic. Its introduction for this purpose was due to the accidental discovery that digitalis in full doses caused contraction of the uterus and therefore checked metrorrhagia. Thus, as in the case of ergot, its use became extended as a remedy for bæmorrhages from other places, but I think there is little or no evidence that it has any action of this kind. It used to be adrocated in the treatment of hæmoptysis on theoretical grounds, digitalis contracting, it was thought, the small arteries and capillaries, from which it was then supposed that hæmorrhage in hremoptysis took place. As a matter of fact, it was found to do little of the kind, and now we know that its action could not be beneficial, but, if it had any action at all, would probably be harmful, inasmuch as the hæmorrhage comes not from small vessels but from an aneurysm or rupture of a large vessel in the lung, so that if digitalis produced the effect desired and contracted the small arteries it would dam the blood up beyond the ruptured ressel and thus make the hæmorrhage greater. I suppose the giving of digitalis for hæmoptysis would in the early days have been called rational because physiological reason could be assigned, yet, tested at the bedside, it was found to be useless. Now the theory upon which it was based is known to be incorrect, the treatment ceases to be rational and the rational therapeutist does not use it for this purpose any more. In chronic bronchitis its use has been advocated also, but here again, I think, its only benefit is due to its action upon the heart.

In acute pneumonia digitalis was for a long time one of the stock remedies as it was for other fevers. Upon the temperature in such fevers digitalis has no effect, and in large doses it has long been given up as a dangerous and harmful remedy. Yet it is by no means without its use on account of the effect it has upon the heart. It is from the heart that so many cases of fever die and many of them may be saved by a judicious use of this drug. For myself bowever, I prefer the citrate of caffeine, which, associated with strychnia, I believe to be one of the best combinations where the heart is failing in fevers. The effect of digitalis upon the falling sickness is referred to in the early description of the drug given by Culpepper, which I have quoted, and quite recently Dr. Gowers has advocated the use of this drug in epilepsy in combination with belladonna, as it seems to increase the effect of the bromides. A few years ago full doses of the infusion of digitalis were adrocated in delirium tremens. The discovery was made by accident by Dr. Jones of Jersey, to one of whose patients an ounce of the fresh infusion of digitalis was unintentionally given, but so far from doing harm it did good, and subsequent experience led Dr. Jones to advocate this mode of treatment strongly. I have not used it myself much, for most cases of simple delirium tremens get well with general treatment and little medicine. It will be interesting to know what the personal experience of the society is on this point.

There is a general opinion that the best preparation of the drug is an infusion in cold or warm water, but that which is in most common use is the tincture, the only objection to which is that its strength appears to vary somewhat. The different substances obtained chemically from digitalis and included under the term "digitalin" are not popular on account of the uncertainty of their composition and action. There appear to be at least four different glucosides, all of them very poisonous and with a somewhat different action; they occur in the different common forms of digitalin in different proportions : thus Homolle's digitalin consists almost entirely of Schmiedeberg's digitalin; Nalivelli's crystallised digitalin consists, on the other hand, almost entirely of Schmiedeberg's digitoxin. Until the composition and activity of these different digitalins become more constant I think we should do well to abide by the well-tried preparationsviz, the tincture and the infusion.

Digitalis is slowly absorbed and slowly eliminated; it is thus cumulative in action, so that it is wise to watch the effect of the drug. It may be necessary to suspend its action from time to time. Yet this is by no means always necessary, for I have had patients continue to take the tincture of digitalis for months together with nothing but adrantage. However, the ill effects of digitalis may appear
sometimes quite suddenly and without warning. I 
have already referred to the sudden drop which may take place in the pulse, and another symptom produced by the drug is vomiting. The sickness, which is often spoken of as "cardiac vomiting" because it is so often met with in cardiac cases, is in many instances due to the digitalis, for as soon as the drug is stopped the symptom passes away and does not return unless the drug be given again. The influence of idiosyncrasy is as well marked with digitalis as it is with the other drugs I have been speaking of, and in some instances hardly the smallest dose of the drug can be toierated. I do not know, however, of any peculiar effect that idiosyncrasy causes.

Time has not permitted me to consider all the drugs placed upon the list by your committee. The rest must be reserved for other occasions. In preparing this paper I have had to review my own experience with the common remedies and to consider the conclusions to which it leads me. This has proved a task not only interesting but useful, and I trust that others may have found it so too.

Wimpole-street, $\mathrm{W}$.

\section{EIGHTEEN CASES OF INDURATED PSEUDO-CHANCRES.}

By ARThUR SHILlitoe, M.B. CAMb., F.R.C.S. Exg., REGISTRAR TO THE LONDON LOCK HOSPITAL.

I $A M$ indebted for the following examples of this interesting lesion to the observations my father has made on cases occurring in his private practice during the last few years. Mr. Hutchinson, in the London Hospital Reports for 1866, described twelve such cases, which he has preferred to call relapsing chancres. The word "relapsing" seems to imply recurrence in the original site. This is certainly the usual course, but Mr. Hutchinson, in speaking of these lesions in the Lettsomian Lectures for 1886, said: "They occur to those who have had syphilis, and usually, but not invariably, on the site of former chancres." Again, in his work on Syphilis, ${ }^{1}$ in speaking of second infections of syphilis Mr. Hutchinson records the case of a man who came to him with a "well-characterised" chancre "which he made no doubt he had caught by contagion, although it was only nine months since a previous one, and he was not yet rid of his secondary symptoms." The second sore was on quite a different part of the penis. On the same page he quotes a closely resembling case under the care of Mr. Allingham. Mr. Hutchinson says : "I do not know what to make of these cases of second chancres occurring before the first syphilis is cured and not on the site of the original chancre. It is possible, after all, they are only examples of relapsing or perhaps satellite sores."

Again, the word cbancre in the term "relapsing chancres" seems to imply a greater resemblance of these lesions to the initial sore of syphilis than is justified by the course of after events. Microscopically it is, perhaps, impossible to diagnose an indurated pseudo-chancre from the true infecting sore. But from this point all resemblance ceases. Mr. Hutchinson" ${ }^{2}$ says "they never lead to anything in the way of secondlary symptoms." Against this last statement M. Fournier, in the Arclives Générales de Médecine, 1868, says they are often followed by secondary and tertiary symptoms. The examples I have quoted below seem rather to bear out Mr. Hutchinson's statement. 'Those cases in which secondary symptoms occurred after the appearance of the pseudo-chancre were all cases in which the pseudo-chancre appeared early in the disease-that is to say, within a few months of the primary infectionthus leading one to suppose that the presence of a pseudo-chancre influenced in no way the occurrence of secondary symptoms. Bumstead, in the last edition of his book on Venereal Diseases, calls these lesions "recurrent indurations." The same objections seem to me to hold with regard to the word "recurrent" as in the word "relapsing." To have called these new sores pseudo-chancres simply would seem to imply some relation to the soft or noninfecting sore or chancroid, besides omitting the one cardinal symptom, induration, so that I have preferred to

I Hutchinson: Syphilis, 1889, p. 467.

Loc. cit., p. 121. adopt the name given to these lesions by M. Founiernamely, indurated pseudo-chancres.

Case 1. Appearance of indurated pseudo.chancre without any known cause ten montls after original infection.-In June, 1892, this patient had several indurated sores at the corona and some slightly indurated inguinal glands. The sores appeared five weeks after infection, which took place seven weeks previously, during which time he had had no treatment. He was now treated with mercury, and in a month the sores had all healed, leaving some induration. He was slightly salivated. In August there was an ulcer on the lower lip, and also on both tonsils. In November the throat was still red, though not ulcerated, and the patient complained of weakness. In Feb. 18th, 1893, the throat, which had been quite well, was again red and congested. There was a small spot on the penis. In March all the original sores had again become indurated. The patient said there had been no fresh intercourse. The inguinal glands had not re-indurated; there was no eruption and the throat was still congested. In April the induration had almost disappeared, but the patient again complained of feeling weak. In June there was still some slight induration on the left side of the corona, but no other symptoms. In October there were no. symptoms.

CASE 2. Reappearance of indurated pseudo-chancre five times within eighteen months of the primary infection.-In June, 1890, this patient had a sore on the penis, which had been irritated by some caustic application. The inguinal glands were hard and discrete. It was at this time two. weeks since infection. He was given two-grain pills of mercury and chalk three times a day and was readily salivated. In four weeks the sore had healed and the (?) induration was much diminished, and in August the sore and all induration absolutely disappeared. In October the induration in the sore returned and disappeared again in January, 1891 ; there was then no salivation and no other symptoms. In March the induration was as marked as when first seen, and the patient was still taking the mercurial pills. In A pril the induration, which had disappeared, had again returned. In June there were no symptoms. In July the patient stated that he had had intercourse regularly and the induration bad returned. In October the sore was again red and swollen after intercourse. In January, 1892, there were no symptoms. Since the previous June he had been taking potassium iodide. He had eczema badly, to which he had long been liable. In February the eczema improved under arsenic and there were no other symptoms.

CASE 3. Reappearance of indurated pseudo-chancre twice within twelve months of the original infection.-In July, 1892, this patient, aged twenty-six, had a single sore on the glans penis and some indurated inguinal glands. The last intercourse was five or six weeks previously. Mercurial treatment was employed. In August the sore had healed, but the induration and the glands persisted; in September there was ulceration of the left tonsil; in November the throat was well; and in January, 1893, there were no symptoms. In May, however, the left tonsil was again congested and red. The palate had been ulcerated, but by the end of the month there were no symptoms. In June the induration in the old cicatrix and in the glands had returned, but by October the induration had entirely disappeared and there were no other symptoms. In December there was a return of the induration in the old eicatrix; in January, 1894, the induration softened and the patient was much better; in April the induration still persisted, especially in the inguinal glands. In July, contrary to advice, he married. He had lately been taking a mixture containing mexcury and potassium iodide. In March, 1895, there was still some thickening in the old cicatrix and he had patches of psoriasis in several places. He was ordered to try Donovan's solution. In May the psoriasis was not increasing.

CASE 4. Appearance of indurated pseudo-chancre seven years after the primary infection.-In 1878 this patient was treated for an indurated sore and indurated inguinal glands. $\mathrm{He}$ said that he never had any further symptoms. In November, 1885, he had a single indurated sore and rery indurated inguinal glands. The sore appeared one week after infection, which took place a month previously. He was put on mercury. In December everything had cleared up. There were no symptoms. He was instructed to continue the treatment for some time longer. In March, 1889, the patient had had no further symptoms.

CASE 5. Appearance of indurated pseudo-chancre six years after the original infection. -In February, 1885, this patient 